\title{
Combination of edaravone and neural stem cell transplantation repairs injured spinal cord in rats
}

\author{
Y.Y. Song ${ }^{1}$, C.G. Peng ${ }^{2}$ and X.B. Ye ${ }^{1}$ \\ ${ }^{1}$ Department of Burn and Plastic Surgery, Navy General Hospital, Beijing, China \\ ${ }^{2}$ Department of Orthopedics, China-Japan Union Hospital, Jilin University, \\ Changchun, China \\ Corresponding author: X.B. Ye \\ E-mail: yangqi_66@yeah.net / wmf007@yeah.net \\ Genet. Mol. Res. 14 (4): 19136-19143 (2015) \\ Received August 23, 2015 \\ Accepted October 2, 2015 \\ Published December 29, 2015 \\ DOI http://dx.doi.org/10.4238/2015.December.29.23
}

\begin{abstract}
This study sought to observe the effect of the combination of edaravone and neural stem cell (NSC) transplantation on the repair of complete spinal cord transection in rats. Eighty adult female SpragueDawley (SD) rats were used to establish the injury model of complete spinal cord transection at T9. Animals were divided randomly into four groups ( $\mathrm{N}=20$ each): control, edaravone, transplantation, and edaravone + transplantation. The recovery of spinal function was evaluated with the Basso, Beattie, Bresnahan (BBB) rating scale on days 1, 3, and 7 each week after the surgery. After 8 weeks, the BBB scores of the control, edaravone, transplantation, and combination groups were $4.21 \pm 0.11,8.46 \pm 0.1,8.54$ \pm 0.13 , and $11.21 \pm 0.14$, respectively. At 8 weeks after surgery, the spinal cord was collected; the survival and transportation of transplanted cells were observed with PKH-26 labeling, and the regeneration and distribution of spinal nerve fibers with fluorescent-gold (FG) retrograde tracing. Five rats died due to the injury. PKH-26-labeled NSCs had migrated into the spinal cord. A few intact nerve fibers and pyramidal neurons passed the injured area in the transplantation and combination groups. The numbers
\end{abstract}


of PKH-26-labeled cells and FG-labeled nerve fibers were in the order: combination group > edaravone group and transplantation group > control group ( $P<0.05$ for each). Thus, edaravone can enhance the survival and differentiation of NSCs in injured areas; edaravone with NSC transplantation can improve the effectiveness of spinal cord injury repair in rats.

Key words: Spinal injury; Neural stem cell; Edaravone; Injury repair

\section{INTRODUCTION}

The therapy for spinal cord injuries is an active topic in neurological surgery (Carter et al., 2011; Duchossoy et al., 2011; Weaver et al., 2012; Morizane et al., 2012). Recent studies on the mechanism of spinal cord injury suggest that free radicals affect the development of the spinal cord injury by enhancing partial ischemia-reperfusion injury (Sun et al., 2010; He et al., 2010; Yang et al., 2011; Wang et al., 2011). Edaravone is a radical scavenger (Uji et al., 2008; Hisano et al., 2009; Ye et al., 2011; Ono et al., 2011). The current study aimed to observe the effect of edaravone on the repair of rat spinal cord injury and to explore the potential of the combination of edaravone with neural stem cell (NSC) transplantation to treat spinal cord injury.

\section{MATERIAL AND METHODS}

\section{Materials}

Laboratory animals: ten 14-day-old Sprague-Dawley (SD) fetuses and 80 female SD rats (10 weeks old, $250-300 \mathrm{~g}$ ) were provided by the animal research center of Baotou Medical College (China) (animal quality certificate number: SCXX (Inner Mongolia) 20050003).

\section{Experimental methods}

\section{NSC culture and identification}

One SD rat that was 14-16 days pregnant was euthanized by cervical disconnection and sterilized by immersion in $75 \%$ ethanol, and ten fetuses were obtained by cesarean section. Fetal brains were submerged in Dulbecco's modified Eagle's medium (DMEM): Nutrient Mixture F-12 (F12) (Gibco BRL, Gaithersburg, MD, USA) after the meninges and blood vessels were removed. A suspension was made by repeated pipetting. The suspension then was filtered with a 100-100- $\mu \mathrm{m}$ mesh filter and transferred to a culture flask. Epidermal growth factor (EGF, $10 \mu \mathrm{g} / \mathrm{mL}$ ), basic fibroblast growth factor (bFGF, $10 \mu \mathrm{g} / \mathrm{mL}$ ), and N2 additive agents (Sigma, Saint Louis, MO, USA) were added, and the suspended cells were cultured in an incubator with $5 \% \mathrm{CO}_{2}$ at $37^{\circ} \mathrm{C}$. NSC purification was verified by a proportion of neural epithelial stem cell protein $\geq 70 \%$, neural glial acid protein $\leq 10 \%$, and neurofilament microtubulin $\leq 10 \%$. Qualified NSCs were used for the following experiments.

\section{PKH-26 labeling of NSCs}

NSCs were digested with $1 \mathrm{~mL}$ trypsin or EDTA to form a single-cell layer suspension. Then, $2 \times 10^{7}$ cells were transferred to pyramidal centrifuge tubes, washed with serum-free me- 
dium, and centrifuged for $5 \mathrm{~min}$, and the supernatant was discarded. Next, $1 \mathrm{~mL}$ solution $\mathrm{C}$ (Gibco BRL, Gaithersburg, MD, USA) was added to disperse cells without shaking. The PKH-26 (Amersham Pharmacia, Cambridge, England) dye solution $\left(4 \times 10^{6} \mathrm{M}\right)$ was prepared before labeling; $1 \mathrm{~mL}$ resuspended cells was added to $1 \mathrm{~mL}$ dye solution, mixed immediately by pipetting, and cultured for $2-5 \mathrm{~min}$ at $25^{\circ} \mathrm{C}$. The tube was gently inverted regularly to mix thoroughly at $25^{\circ} \mathrm{C}$. One volume of serum or $1 \%$ BSA was added to stop the stain reaction, followed by incubation for $1 \mathrm{~min}$. One volume of serum-containing medium was used to dilute the mixture. Then, the mixed solution was centrifuged at $400 \mathrm{~g}$ for $10 \mathrm{~min}$ at $25^{\circ} \mathrm{C}$, and the supernatant was discarded. The cell pellets were transferred to new tubes and washed three times. Finally, $10 \mathrm{~mL}$ complete medium was added, the tube was centrifuged, and cells were resuspended.

\section{Stem cell transplantation (spinal cord injury, $S \mathrm{Cl}$ ) model and grouping}

Rats $(\mathrm{N}=80)$ were anesthetized with $10 \%$ chloral hydrate $(300 \mathrm{mg} / \mathrm{kg})$ by intraperitoneal injection and fixed on the surgery table facing down. After standard sterilization, an incision was made in the middle of T10 at the back. The T9-T11 vertebral plates were removed to expose the spinal cord, and the model was established with a modified Allen method (Young W 2002) (weight dropping). SCI animals were divided randomly into four groups. The control group $(\mathrm{N}=20)$ was injected with vehicle (PBS) after the injury. In the edaravone group $(\mathrm{N}=20)$, edaravone $(3 \mathrm{mg} / \mathrm{kg})$ was injected through the caudal vein $6 \mathrm{~h}$ after $\mathrm{SCl}$ and then once per day for 1 week. In the transplantation group $(\mathrm{N}=20)$, $6 \mathrm{~h}$ after $\mathrm{SCl}$, the injured spinal cord areas were exposed, and $10 \mu \mathrm{L}$ cell suspension $\left(1 \times 10^{10} / \mathrm{L}\right)$ was injected slowly into the injured area through two ends of the injured cord; the injection was finished within $3 \mathrm{~min}$. The needle remained in the injured area for $5 \mathrm{~min}$ after the injection, and medical biogel was used to seal the injected spot to prevent the outflow of the cell suspension. The opening was sutured layer by layer. In the edaravone + transplantation group $(\mathrm{N}=20), 6 \mathrm{~h}$ after SCl, animals received NSC transplantation and edaravone injection $(3 \mathrm{mg} / \mathrm{kg})$ via the caudal vein. The injection was performed once per day for 1 week. After the surgery, penicillin (30,000 U/kg x 2) was injected intraperitoneally each day for 7 days; animals received bladder massages twice per day to facilitate micturition until the urination reflex recovered (approximately 2 weeks).

\section{Basso, Beattie, Bresnahan (BBB) rating of rear limb motor function}

All SCl rats were filmed and video-recorded in a stable, open environment on days 1, 3, and 7 of weeks 1, 2, 4, 6, and 8 after the surgery. The activity of the rats after surgery was recorded by pictures and videos. BBB function was rated based on the rear limb position, movement methods, and movement range. The BBB rating is a standard rating scale targeting the motor function recovery after spinal cord injury in rats, mainly using the movement range and coordination of rear limbs, tail, and lower torso as indices. The score ranges from 0 for no movement to 21 for normal movement. The scale can indirectly reflect the recovery of an injured spinal cord. The rating was conducted by two independent evaluators who were not involved with the experiment (doubleblinded). The average score of the two was used as the final result to reduce the bias of the score.

\section{Fluorescence microscopy}

Eight weeks after $\mathrm{SCl}$, the injured spinal cord tissues of four randomly selected rats from each group were collected for histological examination to confirm the injury. Samples were fixed 
with $40 \mathrm{~g} / \mathrm{L}$ paraformaldehyde and cut into slices by standard frozen slicing, and the slices were observed under a fluorescent microscope (OLYMPUS IX71, Olympus Optical Co., Ltd., Tokyo, Japan). Ten fields of each slice were selected randomly to count $\mathrm{PKH}-26$-positive cells at high magnification (200X), and the average was used as the number of PKH-26-positive cells of each group.

\section{Fluorescent-gold (FG) retrograde tracing of rat sciatic nerve}

Seven weeks after the surgery, four rats from each group were selected randomly. The animals were anesthetized, and the sciatic nerve was exposed at gaps between the vastus lateralis muscle; the nerve was injured with a tissue forceps. In the dark, $2 \%$ fluorescent-gold (Sigma) was injected at a rate of $0.1 \mu \mathrm{L} / \mathrm{min}$ from many spots within the injured area using a micro-injector; each side received $0.4 \mu \mathrm{L}$. The needle remained at the injured area for 5 min after the injection. Penicillin powder $(80,000 \mathrm{U})$ was dispersed to the opening, and the opening was sutured. Animals were cared for as normal. After 1 week, tissues were collected from the injured area for histological examination. The tissues were submerged in $30 \%$ sugar solution overnight at $4^{\circ} \mathrm{C}$. The next day, samples were embedded with optimal cutting temperature (OCT, Advanced Magnetic, Cambridge, MA, USA) compound and cut into $20-\mu \mathrm{m}$ transections and coronal slices at $-18^{\circ} \mathrm{C}$. Five slices were selected from each animal, and the distribution of FG-labeled cells was observed under a fluorescent microscope (OLYMPUS IX71, Olympus Optical Co., Ltd.).

\section{Statistical analysis}

The SPSS13.0 software (USA) was used for analysis. All data are reported as means \pm standard deviation. Repeated-measures ANOVA and the least significant difference $t$-test were used for multiple comparisons among groups. $\mathrm{P}<0.05$ indicated statistical significance.

\section{RESULTS}

\section{NSC morphology}

One hour after the brain tissue single-cell suspension was cultured in the flask, most cells had precipitated to the bottom of the flask. The cells were round and showed no cellular processes. A few small cell clusters were observed. One day later, the number of NSC clusters had increased, but the sizes of the clusters were still small, the shapes were irregular, and some adhered to the wall. Five days later, the number and size of NSC clusters had increased, and clusters had become round (Figure 1).

\section{BBB rating scale evaluation}

Before the surgery, the rear-limb BBB scores of all rats were 21. After the surgery, all rats became paralyzed in both rear limbs. The surgery wound closed well without infection, and suture stitches came off after 3 weeks. Five rats died (two from the control group during weeks 1 and 2; two from the edaravone group during weeks 1 and 3; and one from the transplantation group during week 1. All deaths were due to hematuresis or bladder rupture). BBB scores were all 0 within 3 days after the surgery. Rats in the four groups showed different degrees of recovery in the rear limbs 2 weeks after the surgery. At 4 weeks, the recovery became faster. The combination group recovered faster than the edaravone and transplantation groups; the rear limbs recovered partial 
load bearing, and the rear claws recovered some functions. The differences were significant $(P$ $<0.05)$. All treatment groups had better recovery than the control group ( $P<0.01$ for each). Six weeks after the surgery, the functional recovery of the three treatment groups entered a plateau. At the end of week 8 , the motion coordination of the front and rear limbs of rats in the combination group recovered, and the palm resumed the load-bearing position but could not lift the body from the table. The motion coordination in the transplantation and edaravone groups was poor, and there was no significant difference between these two groups $(P>0.05$; Table 1$)$.

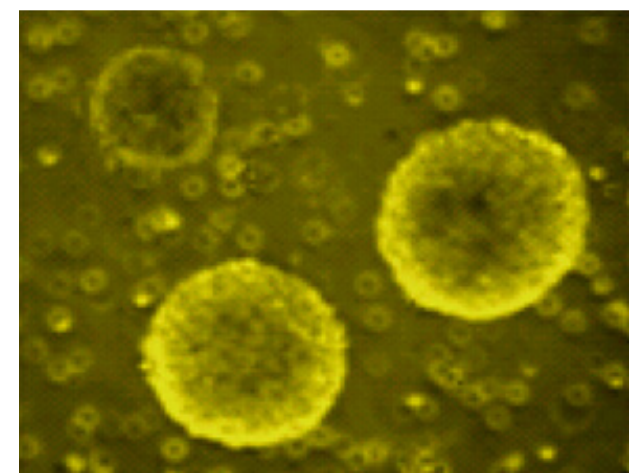

Figure 1. Morphology of primary cultured neural stem cells observed under a microscope (40X).

Table 1. Basso, Beattie, Bresnahan (BBB) score in each group at varying time points after transplantation (means $\pm S D, N=20$ ).

\begin{tabular}{lcccr}
\hline Time & Control group & Edaravone group & NSC group & Edaravone + NSC group \\
\hline 1 day & 0 & 0 & 0 & 0 \\
3 days & 0 & 0 & 0 & 0 \\
1 week & $0.60 \pm 0.11$ & $1.27 \pm 0.09$ & $1.33 \pm 0.10$ & $2.21 \pm 0.1$ \\
2 weeks & $1.34 \pm 0.12$ & $3.25 \pm 0.11$ & $3.41 \pm 0.10$ & $3.75 \pm 0.11$ \\
4 weeks & $2.41 \pm 0.13$ & $7.42 \pm 0.12$ & $7.76 \pm 0.13$ & $8.21 \pm 0.14$ \\
6 weeks & $3.73 \pm 0.12$ & $8.31 \pm 0.12$ & $8.42 \pm 0.14$ & $10.53 \pm 0.13$ \\
8 weeks & $4.21 \pm 0.11$ & $8.46 \pm 0.10$ & $8.54 \pm 0.13$ & $11.21 \pm 0.14$ \\
\hline
\end{tabular}

\section{Observation by fluorescence microscopy}

Eight weeks after SCl, PKH-26-stained red fluorescence was observed in slices of the transplantation and combination groups. The control group had $0 \pm 0.00 \mathrm{PKH}-26$-positive cells per highmagnification field (Figure 2A), the edaravone group $0 \pm 0.00$ (Figure 2B), the NSC group $31.58 \pm 7.64$ (Figure $2 \mathrm{C}$ ), and the combination group $68.62 \pm 9.44$ (Figure 2D). The NSC and edaravone groups were significantly different from the control group $(P<0.05)$, as was the combination group $(P<0.01)$.

\section{The FG retrograde cortex-spinal cord tracing}

The numbers of FG-labeled pyramidal cells and axons in the head end of the injured spinal cord area were as follows: control group 9.6 \pm 2.1 (Figure 3A), edaravone group $24.6 \pm 3.6$ (Figure 3B), NSC group $29.4 \pm 4.0$ (Figure 3C), and combination group $42.6 \pm 5.1$ (Figure 3D). The NSC and edaravone groups were significantly different from control $(P<0.05)$, as was the combination group $(P<0.01)$. 


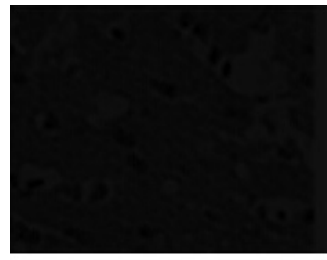

A

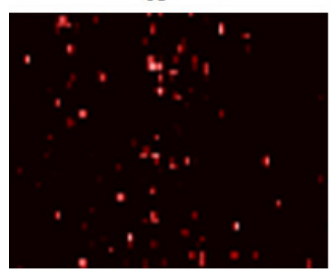

C

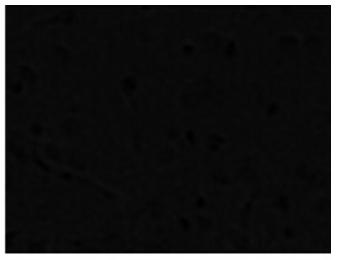

B

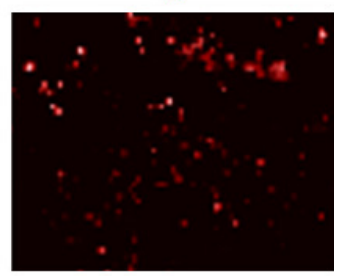

D

Figure 2. A. No PKH-26-positive cells were observed in frozen sections from the control group (200X). B. No PKH-26positive cells were observed in frozen sections from the edaravone group (200X). C. There were less PKH-26-positive cells in the NSC group than in the combination group but more than in the control and edaravone groups (200X). D. $\mathrm{PKH}-26-$ positive cells were widely distributed in frozen sections from the combination group (200X).

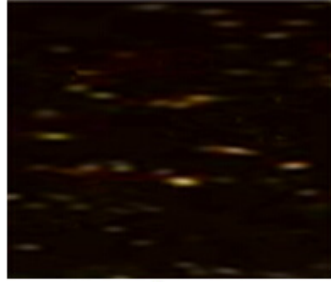

A

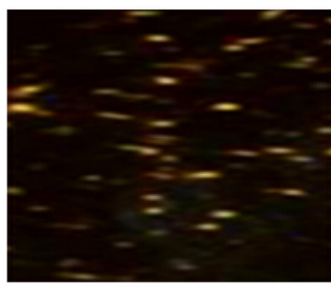

$\mathrm{C}$

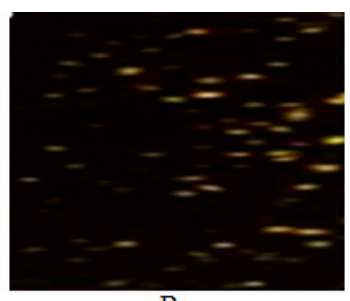

B

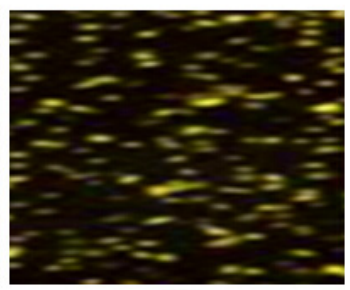

$\mathrm{D}$

Figure 3. A. Very few FG-labeled pyramidal cells and axons were observed in the head end of the injured area in the control group (200X). B. A few FG-labeled pyramidal cells and axons were observed in the head end of the injured area in the edaravone group (200X). C. A few FG-labeled pyramidal cells and axons were observed in the head end of the injured area in the NSC group (200X). D. Many FG-labeled pyramidal cells and axons (golden yellow) were observed in the head end of the injured area in the combination group (200X).

\section{DISCUSSION}

Spinal cord injury is a common pathology in neurosurgery, with a poor prognosis. With the advancements in NSC technology, NSC transplantation has become a new approach to explore the treatment for nervous system injuries. The mechanisms of its treatment of spinal cord injury may be the following: Substitution: implanted NSCs can substitute for lost neurons in the injured sections 
and form connections with axons to restore the conduction pathway (Liu et al., 2000); treatment of spinal cord injury by NSC transplantation occurs mainly through the substitution effect. Support and bridge: transplanted NSCs produce multiple extracellular matrices to fill the space left by injured tissues and provide supportive mass for axon regeneration. Meanwhile, after transplantation to injured spinal cord areas, NSCs differentiate into neurons with different functions, which bridge the broken spinal cord and restore the nerve conduction pathway (Blits et al., 2005). Neurotrophic effect: neurons and glia differentiated from NSCs secrete multiple neurotrophic factors, which improve the microenvironment of the injured tissues and initiate expression of regeneration-related genes, stimulating the regeneration of axons (Kamei et al., 2004; Nakamura et al., 2005). Myelin sheath regeneration effect: NSCs prevent the broken axons of host neurons from retrograding and necrosis and enable the generation of new myelin sheath in the remaining and newly formed nerve fibers (Dasari et al., 2007), maintaining the integrity of nerve fiber functions.

NSC transplantation brings new hope for the treatment of spinal cord injury. However, NSC transplantation alone cannot restore the injured spinal tissue. It is necessary to improve the microenvironment of the injured area to enhance the survival, proliferation, and differentiation of transplanted cells (Lü et al., 2009; Wang et al., 2010; Foret et al., 2010; Liu et al., 2011). Edaravone is a radical scavenger that inhibits oxidative damage to neurons and endothelial cells by eliminating free radicals and inhibiting lipid peroxidation and, therefore, reduces the edema of injured nerve tissues and improves the microenvironment of injured areas. The current study combined edaravone with NSC transplantation to treat spinal cord injury in rats. Our results showed that the numbers of $\mathrm{PKH}-26$-positive cells and FG-positive fibers in the NSC group, edaravone group, and combination group were significantly larger than those in the control group. $\mathrm{PKH}-26$-positive cells were detected in the injured spinal cord 8 weeks after the injury, indicating that implanted NSCs can survive and move within the injured area for a long time. The number of FG-positive fibers indicates the degree of restoration of the transportation function of the axoplasm through nerve fibers and was consistent with the observed rear-limb functional BBB scores. That is, the combination group had better rear-limb function than the NSC, edaravone, and control groups.

These results demonstrate that NSCs can survive, migrate, and form new nerve fibers in the host. The combination of edaravone injected through the caudal vein and NSC transplantation can enhance the survival and differentiation of transplanted cells into neurons. This study provides a new direction for the treatment of spinal cord injury.

\section{REFERENCES}

Blits B, Kitay BM, Farahvar A, Caperton CV, et al. (2005). Lentiviral vector-mediated transduction of neural progenitor cells before implantation into injured spinal cord and brain to detect their migration, deliver neurotrophic factors and repair tissue. Restor. Neurol. Neurosci. 23: 313-324.

Carter LM, McMahon SB and Bradbury EJ (2011). Delayed treatment with Chondroitinase ABC reverses chronic atrophy of rubrospinal neurons following spinal cord injury. Exp. Neurol. 228: 149-156.

Dasari VR, Spomar DG, Gondi CS, Sloffer CA, et al. (2007). Axonal remyelination by cord blood stem cells after spinal cord injury. J. Neurotrauma. 24: 391-410.

Duchossoy Y, David S, Baulieu EE and Robel P (2011). Treatment of experimental spinal cord injury with 3ß-methoxypregnenolone. Brain Res. 1403: 57-66.

Foret A, Quertainmont R, Botman O, Bouhy D, et al. (2010). Stem cells in the adult rat spinal cord: plasticity after injury and treadmill training exercise. J. Neurochem. 112: 762-772.

He S, Zhang WQ, Zhu J, et al. (2010) Rhesus suifukang after spinal cord injury radical regulation changes. Chang Chun Zhong Yi Yao Da Xue Xue Bao 26: 332-333.

Hisano K, Watanabe M and Morimoto $Y$ (2009). Protective effects of the free radical scavenger edaravone against glutamate neurotoxicity in nearly pure neuronal culture. J. Anesth. 23: 363-369. 
Kamei N, Oishi Y, Tanaka N, Ishida O, et al. (2004). Neural progenitor cells promote corticospinal axon growth in organotypic co-cultures. Neuroreport. 15: 2579-2583.

Liu N, Hu DL and Xu T (2011). Neural stem cell transplantation in the treatment of spinal cord injury. Zhong Guo Zu Zhi Gong Cheng Yan Jiu Yu Lin Chuang Kang Fu. 15: 1809-1813.

Liu S, Qu Y, Stewart TJ, Howard MJ, et al. (2000). Embryonic stem cells differentiate into oligodendrocytes and myelinate in culture and after spinal cord transplantation. Proc. Natl. Acad. Sci. U. S. A. 97: 6126-6131.

Lü HZ and Hu JG (2009). Expression of bone morphogenetic proteins-2/4 in neural stem cells and their lineages. Acta. Neurobiol. Exp. (Wars). 69: 441-447.

Morizane K, Ogata T, Morino T, Horiuchi H, et al. (2012). A novel thermoelectric cooling device using Peltier modules for inducing local hypothermia of the spinal cord: the effect of local electrically controlled cooling for the treatment of spinal cord injuries in conscious rats. Neurosci. Res. 72: 279-282.

Nakamura M, Toyama Y and Okano H (2005). Transplantation of neural stem cells for spinal cord injury. Rinsho Shinkeigaku. 45: 874-876.

Ono H, Nishijima Y, Adachi N, Tachibana S, et al. (2011). Improved brain MRI indices in the acute brain stem infarct sites treated with hydroxyl radical scavengers, edaravone and hydrogen, as compared to edaravone alone. A non-controlled study. Med. Gas. Res. 1:12.

Sun YM, Wang QP, Lin FG, et al. (2010). Hyperbaric oxygen treatment of spinal cord injury in rats and its lipid peroxidation reactions. Zhong Hua Wu Li Yi Xue Yu Kang Fu Za Zhi. 9: 654-656.

Uji Y, Yamamoto H, Mori T, Akabori H, et al. (2008). Edaravone improves the survival of rats subjected to hemorrhagic shock without resuscitation. Surg. Today. 38: 476-477.

Wang D, Zhang JJ and Yang ZX (2010). Treatment of spinal cord injury by transplanting neural stem cells with NgR gene silencing. Zhongguo Wei Zhong Bing Ji Jiu Yi Xue. 22: 28-31.

Wang Q, Chen Q, Ding Q, Yang Q, et al. (2011). Sevoflurane postconditioning attenuates spinal cord reperfusion injury through free radicals-mediated up-regulation of antioxidant enzymes in rabbits. J. Surg. Res. 169: 292-300.

Weaver LC, Dekaban GA and Brown A (2012). Anti-CD11d monoclonal antibody treatment for rat spinal cord compression injury. Exp. Neurol. 233: 612-614.

Yang JD, Li GF, Wang JC, et al. (2011). Effects of methylprednisolone on oxygen free radicals in rats with spinal cord injury. Zhong Hua Shi Yong Zhen Duan Yu Zhi Liao Za Zhi. 25: 979-980.

Ye R, Kong X, Yang Q, Zhang Y, et al. (2011). Ginsenoside rd in experimental stroke: superior neuroprotective efficacy with a wide therapeutic window. Neurotherapeutics 8: 515-525.

Young W (2002). Spinal cord contusion models. Prog. Brain. Res.137: 231-255. 